\title{
mRNA Vaccine-Induced Antibodies More Effective than Natural Immunity in Neutralizing SARS-CoV-2 and its High Affinity Variants
}

\section{Yunkai Yu}

National Cancer Institute

Dominic Esposito

Frederick National Laboratory for Cancer Research

Zhigang Kang

National Cancer Institute

Jianming Lu

Codex BioSolutions, Inc.

\section{Alan Remaley}

National Heart Lung and Blood Institute

\section{Leonard Chen}

National Institutes of Health Clinical Center

Valeria De Giorgi

National Institutes of Health Clinical Center

Kamille West

National Institutes of Health Clinical Center

Liang Cao ( $D$ caoli@mail.nih.gov )

National Cancer Institute

\section{Research Article}

Keywords: SARS-CoV-2, angiotensin-converting enzyme, COVID-19, mRNA vaccination

Posted Date: July 21st, 2021

DOI: https://doi.org/10.21203/rs.3.rs-659065/v1

License: (c) (1) This work is licensed under a Creative Commons Attribution 4.0 International License. Read Full License

Version of Record: A version of this preprint was published at Scientific Reports on February 16th, 2022. See the published version at https://doi.org/10.1038/s41598-022-06629-2. 

mRNA vaccine-induced antibodies more effective than natural immunity in neutralizing SARS-CoV-2 and its high affinity variants

Authors: Yunkai $\mathrm{Yu}^{1}$, Dominic Esposito ${ }^{2}$, Zhigang Kang ${ }^{1}$, Jianming Lư ${ }^{3}$, Alan T Remaley ${ }^{4}$, Valeria De Giorgi ${ }^{5}$, Leonard N. Chen ${ }^{5}$, Kamille West ${ }^{5}$, Liang Cao ${ }^{1 *}$

\author{
Affiliations: \\ ${ }^{1}$ Genetics Branch, National Cancer Institute, Bethesda, MD, USA \\ ${ }^{2}$ Protein Expression Laboratory, NCI RAS Initiative, Frederick National Laboratory for Cancer \\ Research, Frederick, MD, USA \\ ${ }^{3}$ Codex BioSolutions, Inc, Gaithersburg, MD, USA \\ ${ }^{4}$ Lipoprotein Metabolism Section, National Heat, Lung, and Blood Institute, Bethesda, MD, USA \\ ${ }^{5}$ Department of Transfusion Medicine, National Institutes of Health Clinical Center, Bethesda, \\ MD, USA
}

Research support: This project was supported by the Intramural Research Program of the National Institute of Health, National Cancer Institute (NCI), and Center for Cancer Research

Correspondence to: Liang Cao, Genetics Branch, Center for Cancer Research, National Cancer Institute, 37 Convent Dr. MSC 4265, Bldg 37, Rm 6134, Bethesda, MD 20892. Phone: (240)

760-6893; E-mail: caoli@,mail.nih.gov

Disclaimers: None 


\begin{abstract}
Several variants of SARS-CoV-2 have emerged. Those with mutations in the angiotensinconverting enzyme (ACE2) receptor binding domain (RBD) are associated with increased transmission and severity. In this study, we developed both antibody quantification and functional assays. Analyses of both COVID-19 convalescent and diagnostic cohorts strongly support the use of RBD antibody levels as an excellent surrogate to biochemical neutralization activities. Data further revealed that the samples from mRNA vaccinated individuals had a median of 17 times higher RBD antibody levels and a similar degree of increased neutralization activities against RBD-ACE2 binding than those from natural infections. Our data showed that N501Y RBD had 5-fold higher ACE2 binding than the original variant. While antisera from naturally infected subjects had substantially reduced neutralization ability against N501Y RBD, all blood samples from vaccinated individuals were highly effective in neutralizing it. Thus, our data indicates that mRNA vaccination is far more effective than natural immunity in generating highly effective neutralizing antibodies. It further suggests a potential need to maintain high RBD antibody levels to control the more infectious SARS-CoV-2 variants.
\end{abstract}

\title{
Introduction
}

Humoral immunity against SARS-CoV-2 can be achieved with either natural infection or vaccination. Most people who had COVID-19 developed sustained serological responses ${ }^{1-4}$. While the antibodies can be detected in most SARS-CoV-2 infected individuals, the antibody levels are highly variable 5 . On the other hand, some of the current assays have limitations in detecting either circulating antibodies ${ }^{5}$ or neutralizing activities ${ }^{2-4}$ in those individuals. In one instance, those with IgG antibody results were semi-quantitative and those with titers of less than 
1:320 would fail to produce detectable neutralizing activities ${ }^{3}$. There are many studies reporting reinfection by SARS-CoV-2 ${ }^{6-9}$, whereas in some of the studies, the neutralizing antibodies were shown to have a protective role ${ }^{10,11}$. In one recent report, individuals who were SARS-CoV-2 seropositive from prior exposure had an estimated $80 \%$ reduction of subsequent risk for reinfection ${ }^{12}$. Several late stage clinical studies demonstrated the effectiveness of COVID-19 vaccines $^{13-17}$. The mRNA-based vaccines, in particular, have achieved remarkable clinical efficacy in protecting the vaccinated subjects against COVID-1913-15. A previous phase 1 study showed that neutralizing activity elicited by Moderna's mRNA vaccine was in the upper half of that of convalescent plasma specimens ${ }^{18}$. There are a few urgent issues to be addressed. First, it is important to understand the differences in specific antibodies and neutralization activities between the vaccine acquired and natural immunity against SARS-CoV-2 infections. This information would help to determine the need to vaccinate against SARS-CoV-2 in those with natural immunity, especially against the variants. Second, as there are many SARS-CoV-2 vaccines already administered to the general population, it is important to determine and monitor antibody levels and neutralization activities to estimate the durations of protection. While clinical trial outcome is the gold standard for efficacy, these vaccine trials take a long time to execute and are subject to extensive variations, especially with emerging and different SARS-CoV-2 variants, which make comparisons difficult. Thus, effective surrogates would be helpful for their assessment. Third, due to emerging SARS-CoV-2 variants, many with increased affinity to the cell surface ACE2 receptor, it is important to adapt the vaccination strategy accordingly for optimal protection against COVID-19.

The spike (S) protein of the virus binds to ACE2 through its $\mathrm{RBD}^{20,22}$, which is becoming a key research area for public health due to its roles in developing neutralizing antibodies ${ }^{23}$, and 
its mutations in emerging SARS-CoV-2 variants spreading rapidly worldwide. The first of such variants with a D614G mutation at the spike protein was shown to increase viral titer and infectivity, yet it was effectively neutralized by antisera ${ }^{24}$. More recently, a UK variant B.1.1.7 was implicated to cause a surge in COVID-19 $\operatorname{cases}^{25}$. It had a mutation N501Y in the RBD region that is directly involved in contacting $\mathrm{ACE} 2^{26}$. N501Y and two other mutations in the RBD domain, K417N/T and E484K, were subsequently founds in SARS-CoV-2 variants from South Africa (B.1.351) ${ }^{27}$ and Brazil (P1) ${ }^{28}$. The N501Y was of particular interest due to its presence in all three variants and its unique role in mediating a direct contact with ACE2 receptor. Mutational scanning studies of SARS-CoV-2 RBD domain in yeast showed the N501F mutation resulted in several fold increased affinity to ACE2 ${ }^{26}$. In a mouse model of SARS-CoV2, the N501Y mutation emerged and conferred increased affinity towards mouse ACE2 receptor $^{29}$. While several recent studies suggested of vaccine antisera that bind and neutralize this B.1.1.7 variant ${ }^{30-33}$, there is a need to investigate the antibody levels after vaccination and protection against infections. With over 150 million COVID-19 cases worldwide by May 2021, and difference vaccines available, it is also important to understand the differences between vaccination and natural immunity, between vaccines, and to better predict the ability of acquired immunity to protect the subjects against emerging variants. Thus, we chose the original and N501Y RBD that appeared in B.1.1.7 and other SARS-CoV-2 variants for the investigation.

\section{Results}

mRNA vaccination induces higher anti-RBD antibody levels than natural immunity against SARS-CoV-2 
Many of the current serology tests for RBD have limitations on sensitivity, dynamic range, and unprecise or semi-quantitative $\mathrm{e}^{3,34,35}$. To accurately determine the levels of anti-RBD antibody in SARS-CoV-2 infected individuals, we developed an electrochemiluminescencebased serology assay for exceptional sensitivity and dynamic range. The assay is based on two antibody-antigen interactions for maximum specificity (Fig. 1A). We purified recombinant RBD from transiently transfected 293 cells and labeled it with either biotin for antibody capture or ruthenium $(\mathrm{Ru})$-tag for antibody detection ${ }^{35}$. Two commercially available RBD monoclonal antibodies (Mab), Mab D001 and D003, were used as the calibrator (D003) and the reference standard (D001) for accurate quantification. Extraordinary large linear quantification range of at least 1,000-fold was achieved with our assay (Supplementary Fig. 1). The assay was subsequently simplified from a three-step to a single incubation step without the loss of performance (Supplementary Fig. 2). In compared with a commercially available spike S1 protein antibody test from Ortho Diagnostics, our RBD antibody test showed strong correlation with it $(r=0.679, P<0.0001)$ (Supplementary Fig. 3). However, our assay exhibited a greater linear dynamic range with COVID sera samples at lower concentrations and improved sensitivity (Supplementary Fig. 3). Thus, our RBD antibody test is quantitative, and has exceptional sensitivity and a large dynamic range.

The clinical validation study of the test was performed using 41 serum samples from 33 convalescent donors with documented history of COVID-19 (Supplementary Table 1) and from 171 healthy donors collected before January 2020. None of the convalescent donors received COVID vaccines. The assay is exceptionally specific, as all negative samples were below of the lower limit of quantification $(\mathrm{N}=171)$. All convalescent donors were positive in the test $(\mathrm{N}=$ 41; Fig. 1B). The median RBD antibody level is $1.3 \mathrm{ug} / \mathrm{mL}$ with a large 170 -fold range for RBD 
antibody levels in this small group of convalescent samples (range, 27-4,800 ng/mL; Fig. 1B). Similar levels of RBD antibody were detected in blood samples taken from those who went to the National Institutes of Health (NIH) Clinical Center for COVID-19 tests and were SARSCoV-2 serology test positive $(\mathrm{N}=39, P=0.973$, Fig. 1B). Interestingly, the blood from donors who completed two doses of mRNA vaccines (Pfizer or Moderna, $N=28$, Supplemental Table 2) had much higher RBD antibody levels than that of the convalescent group (Fig. 1B, $P<$ 0.0001). The median level of RBD antibody for the mRNA vaccine group was $22.3 \mathrm{ug} / \mathrm{mL}$, which was 16.8 -fold higher than $1.3 \mathrm{ug} / \mathrm{mL}$ of the convalescent group.

We examined the RBD antibody levels of the vaccine group and time association and noticed a correlation $(r=-0.522, P=0.004$; Fig. 1C). There was a difference in the antibody levels from samples taken within 2 months and at 6 months post second dose $(P=0.001$; Supplementary Fig. 4). In contrast, convalescent sera did not exhibit a correlative between time and antibody levels, with a median follow-up time of 207 days from the disease onset $(r=0.234$, $P=0.141 ;$ Fig. 1D). The analysis of paired samples from same individuals in the convalescent group showed no change in antibody levels at two different time points $(P=0.396)$, whereas the paring was highly effective ( $r=0.912, P=0.0007$; Supplementary Fig. 5). Hence, the data suggests that the antibody levels of convalescent sera did not decline significantly for at least 8 months post infection, whereas the ultrahigh RBD antibody levels achieved with mRNA vaccines could be subject to a more rapid decline.

\section{Anti-RBD antibody concentration-dependent neutralization against RBD-ACE2 binding with COVID-19 antisera from natural immunity}


To accurately quantify the ability of the antisera in neutralizing RBD and ACE2 binding, we developed an electrochemiluminescence based protein binding assay using recombinant RBD and ACE2 proteins as illustrated in Fig. 2A. When RBD was added to the assay wells, there was an excellent linear relationship between added free RBD and the luminescence signal from the RBD bound to ACE2 $\left(r^{2}=0.99\right.$; Fig. 2B). Therefore, the ACE2 binding assay provides a precise quantification of free RBD capable of binding to ACE2. To clinically validate the sensitivity and specificity of the RBD-ACE2 binding assay, we analyzed the percentage of inhibition with the 41 COVID-19 convalescent sera and a comparable number of pre-COVID-19 control sera. The results showed that the 41 COVID-19 sera had a significantly higher inhibitory effect against RBD-ACE2 binding ( $P<0.0001$; Fig. 2C). The median levels of inhibition were $93 \%$ for the convalescent sera and $7 \%$ for the control sera. When comparing the convalescent sera with the negative controls, the antibody neutralization assay showed high sensitivity and specificity, with an area under curve (AUC) in Receiver Operator Characteristic (ROC) analysis of 0.986 (Supplementary Fig. 6), indicative of high sensitivity and specificity of the assay. The neutralization assay further demonstrated similar consistency when compared with the RBD antibody test using paired samples, with a correlation coefficient for pairing $r=0.952$ that was highly significant $(P=0.0001$; Supplementary Fig. 7$)$. Thus, not only the RBD-ACE2 receptor neutralization assay has excellent sensitivity and specificity for COVID-19 antisera, but also is precise with paired convalescent serum samples taken at different time points.

From the eight donors with paired samples, it is also apparent that those with high antiRBD levels $(>1,000 \mathrm{ng} / \mathrm{mL})$ showed stronger neutralization activity, whereas those with low anti-RBD levels $(<100 \mathrm{ng} / \mathrm{mL})$ showed much lower neutralization activity (Supplementary Fig. 7). The complete analysis of COVID-19 convalescent sera for neutralization activity against 
RBD-ACE2 binding was performed. The results indicated that the neutralization assay for RBDACE2 binding was specific, as $5,000 \mathrm{ng} / \mathrm{mL}$ of the calibrator monoclonal antibody D003, which bound to RBD well, yet exhibited no neutralization activity against RBD-ACE2 binding (Fig. 2D, data point shown with an arrow). As expected, there was an association between the antiRBD antibody level and neutralization activity against RBD-ACE2 binding (correlative analysis, $P<0.0001 ;$ Fig. 2D). Further, the data demonstrated an extraordinary antibody dose-dependent neutralization activity using the nonlinear regression model with $r^{2}=0.928$. The dilution adjusted (6-fold) and estimated 50\% inhibitory concentration inhibition ( $\left.\mathrm{IC}_{50}\right)$, based on the analyses of 41 samples, was $69 \mathrm{ng} / \mathrm{mL}$. RBD antibody concentration-dependent inhibition of RBD-ACE binding was confirmed with the second group of diagnostic serum samples (Supplementary Fig. 8). Thus, there is a strong RBD antibody concentration-dependent neutralization activity against ACE2 binding with two sets of COVID-19 antisera.

\section{N501Y RBD significantly increased ACE2 binding and attenuated the neutralization ability of COVID-19 convalescent antisera}

To investigate the ability of antisera from convalescent patient to recognize the B.1.1.7 N501Y variant, we purified this mutant RBD protein and labeled it with the Ru-tag for electrochemiluminescence assay. We derived a scheme to compare the levels of antibodies against either the WT or N501Y RBD in the same samples (Supplementary Fig. 9). The results with the convalescent donor blood samples showed strong linear correlation between antibodies recognizing both the WT and the N501Y proteins $\left(r^{2}=0.927\right)$. Best-fit analysis revealed a slope of 1.09 (95\% CI, 0.99-1.19; Fig. 3A), suggesting the antisera from COVID-19 convalescent donors bind both the WT and N501Y RBD proteins equally well. 
We further analyzed the ability of COVID-19 convalescent antisera to neutralize the binding of the N501Y RBD to ACE2, as in the case of the WT RBD. The results showed the specific neutralization of the antisera from COVID-19 convalescent donors when compared with that of the pre-COVID-19 donor sera samples ( $P<0.0001$; Fig. 3B). The neutralization assay against RBD-N501Y and ACE2 binding was both very sensitive and specific with AUC of ROC analysis of 0.948 (Supplementary Fig. 10). There was further a strong linear correlation between neutralization activity against the WT and the N501Y RBD in ACE2 binding with a slope of 1.03 $\left(r^{2}=0.896, \mathrm{n}=41\right.$; Supplementary Fig. 11$)$. Thus, COVID-19 antisera neutralize WT and N501Y RBD with an equal potency.

However, we observed dramatic differences in the ability of the WT and N501Y RBD to bind ACE2. Results from five consecutive experiments showed that N501Y RBD bound to ACE2 at an average of 5.1-fold higher rate than the WT RBD (range, 4.1 to 6.1 -fold). A representative result is shown (Supplementary Fig. 12). Thus, it can be concluded that N501Y RBD has a much higher affinity to ACE2. We further examined the absolute level of the WT and the N501Y RBD bound to ACE2 in the presence of COVID-19 antisera. While the antisera neutralized both the WT and N501Y RBD at a similar rate, there was four times (slope $=3.99, \mathrm{~N}$ =41) more N501Y RBD bound to ACE2 in the presence of the convalescent antisera (Fig. 3C). This was further confirmed with the COVID-19 diagnostic sera (Supplementary Fig. 13). As the results of increased ACE2 binding affinity due to N501Y mutation, there were far more absolute amount of N501Y RBD bound to ACE2 than the WT in the presence of COVID-19 convalescent antisera $(P<0.0001, \mathrm{~N}=41$; Fig. 3D). Thus, natural immunity from the original SARS-CoV-2 infections could not consistently provide sufficient neutralization against N501Y RBD variant from binding to the cellular ACE2 receptor. 


\section{mRNA vaccination results in much more effective neutralization than natural immunity against N501Y RBD from binding to ACE2}

To further determine the difference between natural immunity and mRNA vaccination, we selected five samples that had median levels of anti-RDB antibody of each group, and performed dilutions and neutralization studies against N501Y binding to ACE2. The results showed that dilution factors to $\mathrm{IC}_{50}$ were 25.8 and 402.0 for convalescent and mRNA vaccinated blood samples (Fig. 4A, 4B), a difference of 15.6-fold. This difference in neutralization is consistent with that the mRNA vaccinated blood had 16.8-fold higher anti-RBD antibodies than the convalescent blood (Fig. 1B). Thus, the mRNA vaccinated blood is far more effective in neutralizing the high affinity N501Y RBD from binding to ACE2.

The mRNA vaccinated plasma is far more effective in neutralizing N501Y RBD against ACE2 binding when compared to convalescent samples. There were $31.7 \%$ retained over $50 \%$ N501Y RBD and ACE2 binding in the presence of convalescent samples, in contrast to a maximum residual binding of $12.6 \%$ in the presence of mRNA vaccinated samples, a difference that is highly significant $(P<0.0001$; Fig. 4C). Detailed examination of antibody concentration dependent neutralization revealed strong correlations between RBD antibody levels and neutralization activities for both sample groups $\left(r^{2}=0.926\right.$ and $r^{2}=0.823$; Fig. 4D). Thus, the substantially elevated antibody levels in the mRNA vaccine group appeared to be the primary driver of the neutralization activities. Similarly, improved efficacy with the vaccinated blood was also observed against the WT RBD and ACE2 binding (Supplementary Fig. 14). Therefore, the data indicates the insufficiencies of natural immunity, and the superiority of mRNA vaccination 
in neutralizing RBD and ACE2 binding, particularly against SARS-CoV-2 variants with increased affinity to their cell receptor.

\section{Discussion}

In this study, we showed that mRNA vaccinated blood donors have a median of 17 times higher RBD antibody levels when compared with those who became seropositive due to prior COVID-19. Our results indicated an exceptional strong association between high RBD antibody levels in and the ability to biochemically neutralize RBD binding to the cellular ACE2 receptor. The N501Y mutation, while did not alter the neutralizing antibody binding, presented with a 5fold greater affinity to ACE2, which resulted in a drastically reduced ability of COVID-19 convalescent antisera to neutralize its ACE2 binding. Fortunately, the vaccinated blood samples, due to their much elevated RBD antibody levels, were far more effective in neutralizing both the WT and N501Y RBD from binding to ACE. With an average of 16-fold greater potency than convalescent blood, the vaccinated blood samples were more than sufficient to compensate for the 5-fold increased affinity of N501Y RBD, resulting in the highly effective inhibition of both the WT and N501Y RBD from binding to ACE2.

With over 150 million people infected with SARS-CoV-2 by May 2021, one of the critical questions going forward is whether the natural immunity would be sufficient to prevent future reinfections, particularly by more infectious variants. N501Y RBD is central to the investigation as it is the key driver to increased affinity to cell ACE2 receptors. While the reinfections were seen with the original SARS-CoV-2, our results indicated that the antisera from natural immunity would be less effective against variants such as B.1.1.7 due to its increased affinity to ACE2. Thus, many individuals acquired immunity through prior SARS-CoV-2 
infections would not be sufficient to prevent reinfections by new variants, especially in those with low RBD antibody levels.

Our findings would bring advances in the understanding of different vaccines and their abilities to fight off different SARS-CoV-2 variants. There are multiple vaccines tested in various geographic region with different prevalence of SARS-CoV-2 variants. In one study, mRNA vaccine BNT162b2 exhibited 97\% efficacy against symptomatic COVID-19 in Israel dominated by B.1.1.7 variant ${ }^{36}$. Our study showed that not only the mRNA vaccinated plasma has 17-fold higher antibodies than the convalescent antisera, but also 16 time more potential in neutralizing RBD and ACE2 binding of both the original and N501Y mutation that was present in the above studies. Thus, the increased antibody levels were sufficient to compensate for the increase virulence due to higher ACE2 binding of this variant. In another study, an adenovirusbased vaccine ChAdOx1 nCoV-19 exhibited 55\% clinical activity in the $\mathrm{UK}^{16}$ where B.1.1.7 was prevalent. A plausible explanation would be that this vaccine ${ }^{37}$ and another adenovirus vaccine ${ }^{38}$ only produced antibody levels that were comparable to COVID-19 convalescent blood. Thus, the clinical experience with B.1.1.7 suggests that higher levels of RBD antibody would be required to protect the subjects from infections. In the third example, a nanoparticle vaccine NVXCoV2373 was only $50 \%$ effective in South Africa dominated by B.1.351 ${ }^{17}$. The NVX-CoV2373 was be able to induce a 4 -fold increase over convalescent sera ${ }^{39}$. Thought the vaccination works against the difficult B.1.351 variant, there might be a room for further improvement by achieving higher antibody levels.

While there was no trend of decreasing RBD antibodies in those with natural immunity for up to 9 months, our data revealed a large variation of their levels that were stable in given individuals. In comparison, while mRNA vaccines resulted in much higher RBD antibody levels 
than natural infections, this hyper-elevated level appeared to be less stable with samples at 6 months past the second dose. While our work is still very preliminary, it would be interesting to test more cases and over longer duration to see how fast the antibody levels would decline over time, particular in those with hyper-elevated antibody levels.

Likely many other surrogate biomarkers for a medical intervention on a disease, our in vitro receptor-binding neutralization has its limitations. There are factors that cannot be represented in this model. These include the 3D structures of the viral spike protein and ACE2, the surface density of both molecules, the process of viral entry into the cells and more. In addition, the mutation profiles of the variants are drastically simplified in our model. Other RBD mutations, K417N/T and E484K, either alone or in combinations, have not been evaluated in this study. Moreover, the $\mathrm{IC}_{50}$ values that we obtained with in vitro $\mathrm{RBD}-\mathrm{ACE}$ binding assay, while biochemically informative, is not equivalent to an in vivo protective level. Besides, the quantification of RBD antibody levels of antisera is based on the calibration of a monoclonal antibody for consistency and universal adaptability, where the polyclonal nature of the antisera may be simplified. However, the RBD and ACE2 binding is perhaps the most crucial step for early viral pathogenesis, and the RBD variants are of the most concerns for current global health. Our data showed that the protein biochemical neutralization assay is a sensitivity, specificity, quantitative, and reliable biomarker to determine the neutralizing ability of antisera against SARS-CoV-2 and its variants. It could be used immediately as a surrogate for the clinical investigations of SARS-CoV-2 variants and the protection either by natural immunity or vaccination. 


\section{Methods}

\section{Patient or blood donor samples}

Serum samples were collected at convalescent donors with prior documented COVID-19 under the NIH clinical protocol (ClinicalTrials.gov identifier: NCT04360278). A total of 41 samples were prospectively collected from 33 donors who had confirmed COVID-19 (Supplementary Table 1). An additional were collected under the same protocol from 28 people who never had COVID-19 but completed two doses of COVID-19 mRNA vaccines, either Pfizer or Moderna (Supplementary Table 2). All these samples were and processed at a single site within 4 hrs of blood draw in compliance of Good Clinical Practice. The samples were collected using the Institutional Review Board-approved protocol at the US National Institutes of Health with informed consent. Informed consent was obtained from all study participants. All methods were performed in accordance with the relevant guidelines and regulations. Patient samples were de-identified and tested in an unbiased and blinded fashion.

Additional samples from 38 patients were obtained from Department of Laboratory Medicine of NIH as diagnostics samples. The samples were selected based on the positive diagnoses and no personal or medical information was made available at any time for this research.

\section{RBD protein expression and purification}

Recombinant SARS-CoV-2 spike receptor-binding domain (RBD) proteins contained a tissue plasminogen activator (TPA) signal peptide followed by amino acids 318-529 of spike (wild type or N501Y mutant) with a HRV-3C protease site and a C-terminal His(8)-streptavidin binding peptide tag. Proteins were expressed in Expi293 cells (ThermoFisher) for 96 hours at 
$32^{\circ} \mathrm{C}$ and purified as previously described ${ }^{35,40}$. Final proteins were validated by mass spectrometry and size exclusion chromatography.

\section{RBD antibody assay}

The recombinant RBD proteins were labeled with either biotin or MDS ruthenium-tag as previous described ${ }^{41}$. The RBD monoclonal antibodies for calibration (D003) and reference standard (D001) were acquired from Sino Biological (Wayne, PA, USA). For serology assay to determine the antibody levels of human sera, $2.5 \mathrm{uL}$ of serum samples were diluted with diluent to $25 \mathrm{uL}$, mixed with $25 \mathrm{uL}$ of $1 \mathrm{ug} / \mathrm{mL}$ biotin-RBD (capture) and $25 \mathrm{uL}$ of $1 \mathrm{ug} / \mathrm{mL}$ rutheniumRBD (detection). All reagents were added together into 96-well streptavidin-coated assay plates (Meso-Scale Diagnostics, Rockville, MD, USA). Both calibrator and reference standard were added in the place of sera. Incubation was carried out at room temperature for $1 \mathrm{hr}$ with constant shaking. After three washes with $150 \mathrm{uL}$ of wash buffer, $150 \mathrm{uL}$ of $2 \mathrm{X}$ read buffer was added, followed by reading with a Meso-Scale QuickPlex SQ120 within 5 min.

\section{Neutralization assay}

The RBD proteins were labeled with MDS ruthenium-tag as described above. The recombinant human ACE2 (aa 18-740) was expressed in NS0 cells, affinity purified, and biotinylated (R\&D Systems, Minneapolis, MN, USA). The streptavidin-coated was used for the assay, on which $25 \mathrm{uL}$ of $1 \mathrm{ug} / \mathrm{mL}$ biotin-ACE2 were added and incubated at room temperature for $1 \mathrm{hr}$. At the same time, serum neutralization was carried out with $60 \mathrm{ng} / \mathrm{mL}$ ruthenium-tag RBD mixed with $5 \mathrm{uL}$ of human sera in a total of $30 \mathrm{uL}$ (1:6 dilution of sera) and incubated at $37^{\circ} \mathrm{C}$ for $1 \mathrm{hr}$. They were then added to the biotin-ACE2 assay plate for a further $1 \mathrm{hr}$ incubation. 
This is followed by the addition of read buffer and QuickPlex reading. Standard calibration curves were obtained with diluted ruthenium-tag RBD starting from $200 \mathrm{ng} / \mathrm{mL}$ at the highest level without incubating with sera.

\section{Statistical analyses}

Data analyses were performed using GraphPad Prism 8. Mann-Whitney non-parametric t test was used to compare results of two unrelated groups such as the RBD antibody levels of diagnostic and convalescent samples. Paired $t$ test was performed in case of multiple blood samples from the same convalescent donors over time, to evaluate the changes in antibody levels and in neutralization abilities.

Correlative analysis was performed to evaluate the association of medical parameters, such as time to disease onset, age of disease onset, duration, and sex, with the levels of antibody. It was also performed to determine the assay specificity in the serum neutralization assay between the COVID-19 antisera and the pre-COVID-19 controls. ROC analysis was performed to determine the sensitivity and specificity of neutralization assays against the WT and the N501Y RBD proteins.

Four parameter logistic regression model provided by Meso-Scale Discovery Workbench 4.0 was used to determine the concentrations of anti-RBD antibodies in sera. Linear regression analysis with four parameters was performed to determine the linearity of both antibody assay and neutralization assay. It is also used to demonstrate the linear relationship in the activities of antisera in recognizing and in neutralizing both the WT and the N501Y RBD. It was further used to determine the relative ratios of these two proteins in binding to ACE2 in the absence and presence of COVID-19 sera from convalescent donors or diagnostic patients. 
A nonlinear regression model with variable slope and four parameters was used to curve fit inhibitory dose-response data to determine the $\mathrm{IC}_{50}$ values for the neutralization of $\mathrm{RBD}$ in ACE2 binding. As all samples were diluted 6-fold prior to the test, the results in the text were adjusted accordingly from those in the figures.

\section{References}

1 Gudbjartsson, D. F. et al. Humoral Immune Response to SARS-CoV-2 in Iceland. N Engl J Med 383, 1724-1734, doi:10.1056/NEJMoa2026116 (2020).

2 Seow, J. et al. Longitudinal observation and decline of neutralizing antibody responses in the three months following SARS-CoV-2 infection in humans. Nat Microbiol 5, 1598-1607, doi:10.1038/s41564-020-00813-8 (2020).

3 Wajnberg, A. et al. Robust neutralizing antibodies to SARS-CoV-2 infection persist for months. Science 370, 1227-1230, doi:10.1126/science.abd7728 (2020).

4 Crawford, K. H. D. et al. Dynamics of neutralizing antibody titers in the months after SARS-CoV-2 infection. J Infect Dis, doi:10.1093/infdis/jiaa618 (2020).

5 Self, W. H. et al. Decline in SARS-CoV-2 Antibodies After Mild Infection Among Frontline Health Care Personnel in a Multistate Hospital Network - 12 States, April-August 2020. MMWR Morb Mortal Wkly Rep 69, 1762-1766, doi:10.15585/mmwr.mm6947a2 (2020).

6 Chan, P. K. S. et al. Serologic Responses in Healthy Adult with SARS-CoV-2 Reinfection, Hong Kong, August 2020. Emerg Infect Dis 26, 3076-3078, doi:10.3201/eid2612.203833 (2020).

7 Lu, J. et al. Clinical, immunological and virological characterization of COVID-19 patients that test re-positive for SARS-CoV-2 by RT-PCR. EBioMedicine 59, 102960, doi:10.1016/j.ebiom.2020.102960 (2020).

8 Selhorst, P. et al. Symptomatic SARS-CoV-2 reinfection of a health care worker in a Belgian nosocomial outbreak despite primary neutralizing antibody response. Clin Infect Dis, doi:10.1093/cid/ciaa1850 (2020).

9 Van Elslande, J. et al. Symptomatic SARS-CoV-2 reinfection by a phylogenetically distinct strain. Clin Infect Dis, doi:10.1093/cid/ciaa1330 (2020).

10 Lumley, S. F. et al. Antibody Status and Incidence of SARS-CoV-2 Infection in Health Care Workers. N Engl J Med 384, 533-540, doi:10.1056/NEJMoa2034545 (2021).

11 Addetia, A. et al. Neutralizing Antibodies Correlate with Protection from SARS-CoV-2 in Humans during a Fishery Vessel Outbreak with a High Attack Rate. J Clin Microbiol 58, doi:10.1128/JCM.02107-20 (2020).

12 Letizia, A. G. et al. SARS-CoV-2 seropositivity and subsequent infection risk in healthy young adults: a prospective cohort study. Lancet Respir Med, doi:10.1016/S2213-2600(21)00158-2 (2021).

13 Baden, L. R. et al. Efficacy and Safety of the mRNA-1273 SARS-CoV-2 Vaccine. N EnglJ Med 384, 403-416, doi:10.1056/NEJMoa2035389 (2021).

14 Dagan, N. et al. BNT162b2 mRNA Covid-19 Vaccine in a Nationwide Mass Vaccination Setting. $N$ Engl J Med 384, 1412-1423, doi:10.1056/NEJMoa2101765 (2021). 
Polack, F. P. et al. Safety and Efficacy of the BNT162b2 mRNA Covid-19 Vaccine. N Engl J Med 383, 2603-2615, doi:10.1056/NEJMoa2034577 (2020).

16 Voysey, M. et al. Safety and efficacy of the ChAdOx1 nCoV-19 vaccine (AZD1222) against SARSCoV-2: an interim analysis of four randomised controlled trials in Brazil, South Africa, and the UK. Lancet 397, 99-111, doi:10.1016/S0140-6736(20)32661-1 (2021).

17 Shinde, V. et al. Efficacy of NVX-CoV2373 Covid-19 Vaccine against the B.1.351 Variant. N Engl J Med, doi:10.1056/NEJMoa2103055 (2021).

18 Jackson, L. A. et al. An mRNA Vaccine against SARS-CoV-2 - Preliminary Report. N Engl J Med 383, 1920-1931, doi:10.1056/NEJMoa2022483 (2020).

19 Hoffmann, M. et al. SARS-CoV-2 Cell Entry Depends on ACE2 and TMPRSS2 and Is Blocked by a Clinically Proven Protease Inhibitor. Cell 181, 271-280 e278, doi:10.1016/j.cell.2020.02.052 (2020).

20 Walls, A. C. et al. Structure, Function, and Antigenicity of the SARS-CoV-2 Spike Glycoprotein. Cell 183, 1735, doi:10.1016/j.cell.2020.11.032 (2020).

21 Samavati, L. \& Uhal, B. D. ACE2, Much More Than Just a Receptor for SARS-COV-2. Front Cell Infect Microbiol 10, 317, doi:10.3389/fcimb.2020.00317 (2020).

22 Lan, J. et al. Structure of the SARS-CoV-2 spike receptor-binding domain bound to the ACE2 receptor. Nature 581, 215-220, doi:10.1038/s41586-020-2180-5 (2020).

23 Barnes, C. O. et al. SARS-CoV-2 neutralizing antibody structures inform therapeutic strategies. Nature 588, 682-687, doi:10.1038/s41586-020-2852-1 (2020).

24 Hou, Y. J. et al. SARS-CoV-2 D614G variant exhibits efficient replication ex vivo and transmission in vivo. Science 370, 1464-1468, doi:10.1126/science.abe8499 (2020).

25 Kirby, T. New variant of SARS-CoV-2 in UK causes surge of COVID-19. Lancet Respir Med 9, e20e21, doi:10.1016/S2213-2600(21)00005-9 (2021).

26 Starr, T. N. et al. Deep Mutational Scanning of SARS-CoV-2 Receptor Binding Domain Reveals Constraints on Folding and ACE2 Binding. Cell 182, 1295-1310 e1220, doi:10.1016/j.cell.2020.08.012 (2020).

27 Tegally, H. et al. Sixteen novel lineages of SARS-CoV-2 in South Africa. Nat Med, doi:10.1038/s41591-021-01255-3 (2021).

28 Candido, D. S. et al. Evolution and epidemic spread of SARS-CoV-2 in Brazil. Science 369, 12551260, doi:10.1126/science.abd2161 (2020).

$29 \mathrm{Gu}, \mathrm{H}$. J. et al. Adaptation of SARS-CoV-2 in BALB/c mice for testing vaccine efficacy. Science 369, 1603-+, doi:10.1126/science.abc4730 (2020).

30 Rathnasinghe, R. et al. The N501Y mutation in SARS-CoV-2 spike leads to morbidity in obese and aged mice and is neutralized by convalescent and post-vaccination human sera. medRxiv, doi:10.1101/2021.01.19.21249592 (2021).

31 Wang, Z. et al. mRNA vaccine-elicited antibodies to SARS-CoV-2 and circulating variants. bioRxiv, doi:10.1101/2021.01.15.426911 (2021).

$32 \mathrm{Wu}, \mathrm{K}$. et al. mRNA-1273 vaccine induces neutralizing antibodies against spike mutants from global SARS-CoV-2 variants. bioRxiv, doi:10.1101/2021.01.25.427948 (2021).

33 Xie, X. et al. Neutralization of N501Y mutant SARS-CoV-2 by BNT162b2 vaccine-elicited sera. bioRxiv, doi:10.1101/2021.01.07.425740 (2021).

34 Amanat, F. et al. A serological assay to detect SARS-CoV-2 seroconversion in humans. Nat Med 26, 1033-1036, doi:10.1038/s41591-020-0913-5 (2020).

35 Klumpp-Thomas, C. et al. Standardization of ELISA protocols for serosurveys of the SARS-CoV-2 pandemic using clinical and at-home blood sampling. Nat Commun 12, 113, doi:10.1038/s41467020-20383-x (2021). 
Haas, E. J. et al. Impact and effectiveness of mRNA BNT162b2 vaccine against SARS-CoV-2 infections and COVID-19 cases, hospitalisations, and deaths following a nationwide vaccination campaign in Israel: an observational study using national surveillance data. Lancet, doi:10.1016/S0140-6736(21)00947-8 (2021).

37 Folegatti, P. M. et al. Safety and immunogenicity of the ChAdOx1 nCoV-19 vaccine against SARSCoV-2: a preliminary report of a phase $1 / 2$, single-blind, randomised controlled trial. Lancet 396, 467-478, doi:10.1016/S0140-6736(20)31604-4 (2020).

38 Logunov, D. Y. et al. Safety and immunogenicity of an rAd26 and rAd5 vector-based heterologous prime-boost COVID-19 vaccine in two formulations: two open, non-randomised phase 1/2 studies from Russia. Lancet 396, 887-897, doi:10.1016/S0140-6736(20)31866-3 (2020).

39 Keech, C. et al. Phase 1-2 Trial of a SARS-CoV-2 Recombinant Spike Protein Nanoparticle Vaccine. N Engl J Med 383, 2320-2332, doi:10.1056/NEJMoa2026920 (2020).

40 Mehalko, J. et al. Improved production of SARS-CoV-2 spike receptor-binding domain (RBD) for serology assays. Protein Expr Purif 179, 105802, doi:10.1016/j.pep.2020.105802 (2021).

$41 \mathrm{Yu}, \mathrm{Y}$. et al. Elevated Serum Megakaryocyte Potentiating Factor as a Predictor of Poor Survival in Patients with Mesothelioma and Primary Lung Cancer. J Appl Lab Med 3, 166-177, doi:10.1373/jalm.2017.025015 (2018).

Acknowledgements: We are grateful for all blood donors and patients for participating the clinical studies at the National Institute of Health (NIH), USA. We thank Matthew Drew, Kelly Snead, Jennifer Mehalko, and Vanessa Wall of the Frederick National Laboratory for production of recombinant RBD proteins. This project has been funded by Federal grants for Intramural Research Program of the NIH, National Cancer Institute, NIH Clinical Center, National Heart, Lung, and Blood Institute, and with Federal fund from the National Cancer Institute under contract number HHSN261200800001E.

\section{Author contributions}

Conceptualization: KW, LC

Methodology: YY, DE, ZK, JL

Patient samples: LNC, KW, ATR, VDG

Supervision: KW, LC

Writing: DE, ZK, AR, LNC, VDG, KW, LC

Reviewing: all authors 
Additional Information: All authors declare no competing interests.

\section{Figure Legends}

Fig. 1. COVID-19 serology assay and antibody levels. (A) Diagram of the serology assay using electrochemiluminescence detection method. The capture RBD protein was attached to the assay plate via biotin and the detection RBD was conjugated to the voltage-sensitive ruthenium (Ru) molecule to emit light. (B) Quantitative RBD antibody data from sera samples of three cohorts: 1) samples on the first seropositive test, 2) convalescent donors with documented COVID-19, 3) donors without prior COVID-19 and completed two doses of mRNA vaccines. Negative control samples were collected from healthy donors prior to Jan. 2020. (C) The RBD antibody levels and time association in vaccinated donors $(P=0.0044)$. (D) The RBD antibody levels and time association in COVID-19 convalescent donors $(P=0.141)$.

Fig. 2. Neutralization assay, sensitivity, specificity, stability, and association with RBD antibody levels in COVID-19 convalescent sera. (A) Diagram of RBD and ACE2 biding assay and serum neutralization assay, where the neutralizing antibody prevents RBD from binding to ACE2. (B) Linearity of the RBD-ACE2 binding assay where increased RBD results in linear increase of binding signal $\left(r^{2}=0.990\right)$. (C) Neutralization data showing the convalescent sera have much higher neutralizing ability $(P<0.0001)$. (D) Serum neutralization assay results using COVID-19 convalescent sera showing strong correlation between anti-RBD levels and neutralizing activity $(P<0.0001, \mathrm{~N}=41)$. The inhibitory concentration $\left(\mathrm{IC}_{50}\right)$ shown was determined using nonlinear regression inhibitory model using RBD antibody levels in the sera, without accounting for a 6-fold dilution. 
Fig. 3. Comparison of antibody levels and neutralization activities against both the wildtype RBD and N501Y RBD proteins with convalescent sera. (A) Linearity of the antiWT-RDB and anti-N501Y-RBD detected in the convalescent sera with a slope of $1.087\left(r^{2}=\right.$ 0.927). (B) Neutralization data from COVID-19 convalescent sera and negative controls. The convalescent sera had specific neutralizing activity against N501Y-RBD $(P<0.0001)$. (C) Linear regression analysis of the ACE2 bound the WT and N501Y RBD detected using the COVID-19 convalescent sera with a slope of $3.99\left(r^{2}=0.896, \mathrm{~N}=41\right)$. (D) N501Y RBD have much higher absolute ACE2 binding than the WT RBD in the presence of neutralizing convalescent sera $(P<$ $0.0001, \mathrm{~N}=41)$.

Fig. 4. mRNA vaccine far more effective than natural immunity in neutralizing N501Y RBD mutant in ACE2 binding. (A) Dilution study to determine the $\mathrm{IC}_{50}$ of representative convalescent samples with median levels of anti-RBD antibodies. (B) Dilution study to determine the $\mathrm{IC}_{50}$ of representative vaccinated samples with median levels of anti-RBD antibodies. (C) Vaccinated blood samples more effective than convalescent ones to inhibit N501Y RBD in ACE2 binding $(P<0.0001)$. (D) Antibody concentration-dependent inhibition of N501Y RBD and ACE2 binding with blood samples from natural immunity $\left(r^{2}=0.926\right)$ and mRNA vaccination $\left(r^{2}=0.823\right)$. 
A

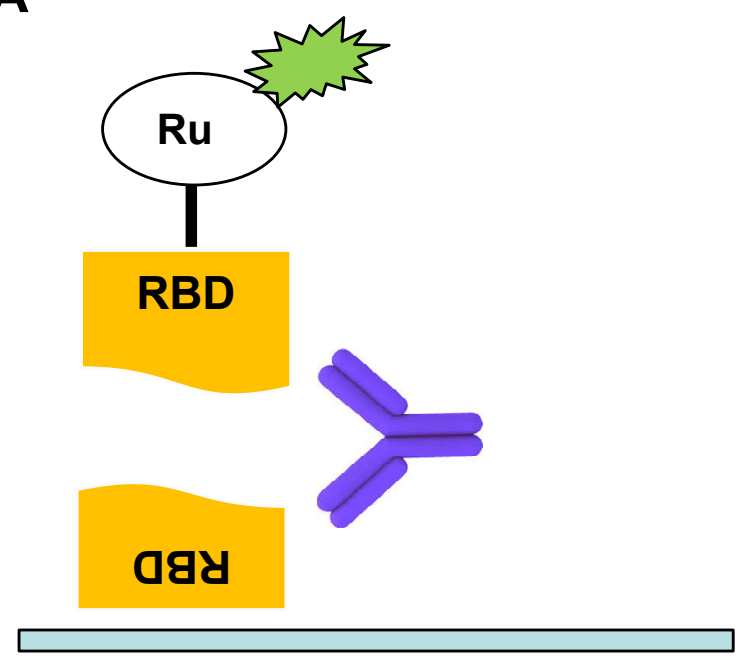

C

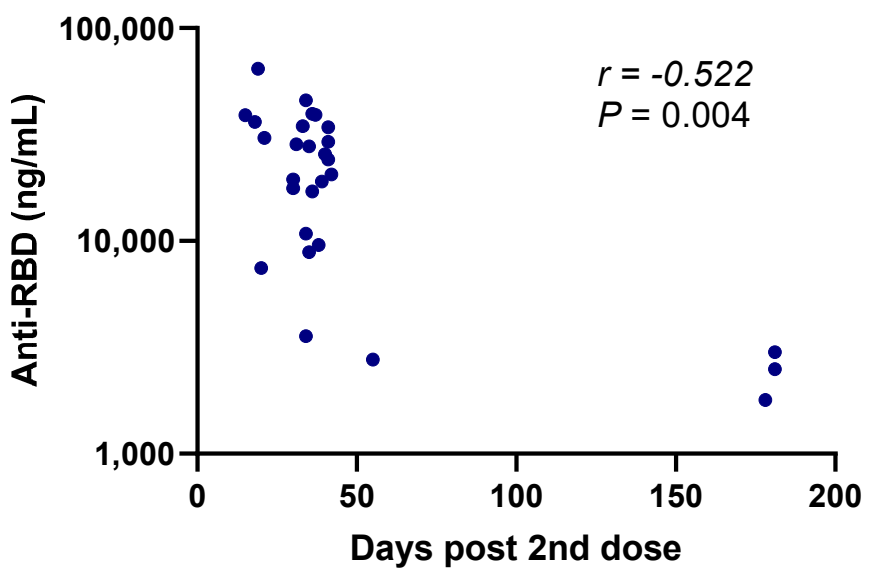

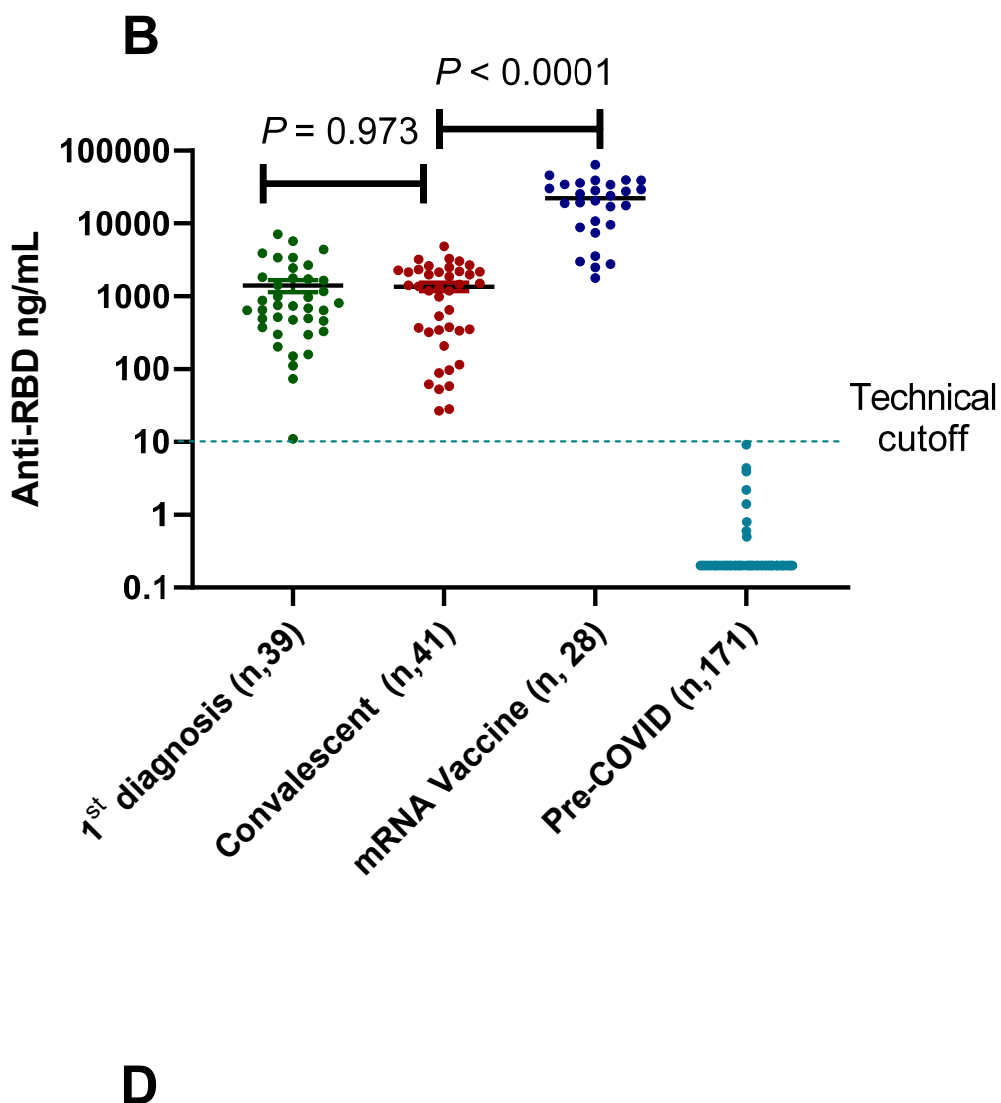

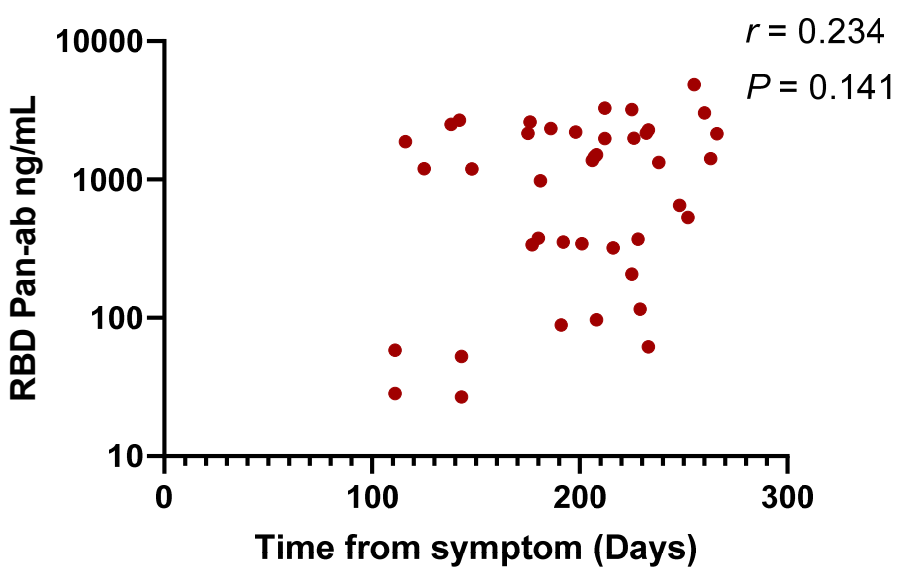


A

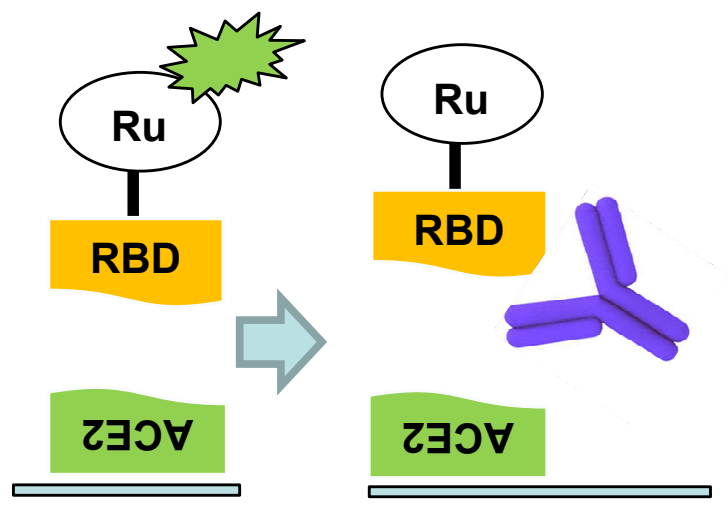

C

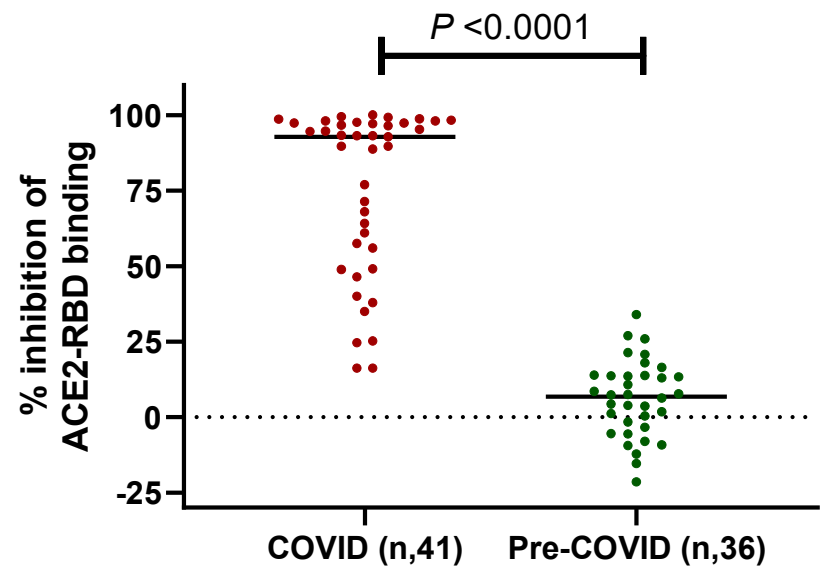

B

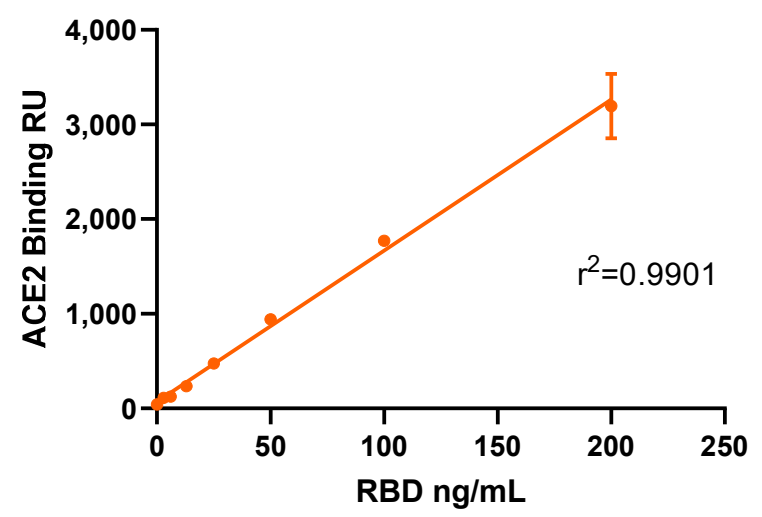

D

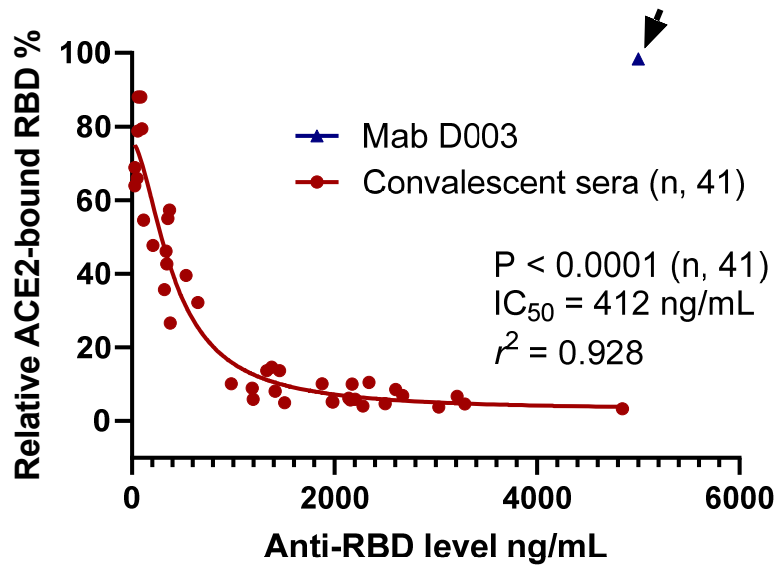




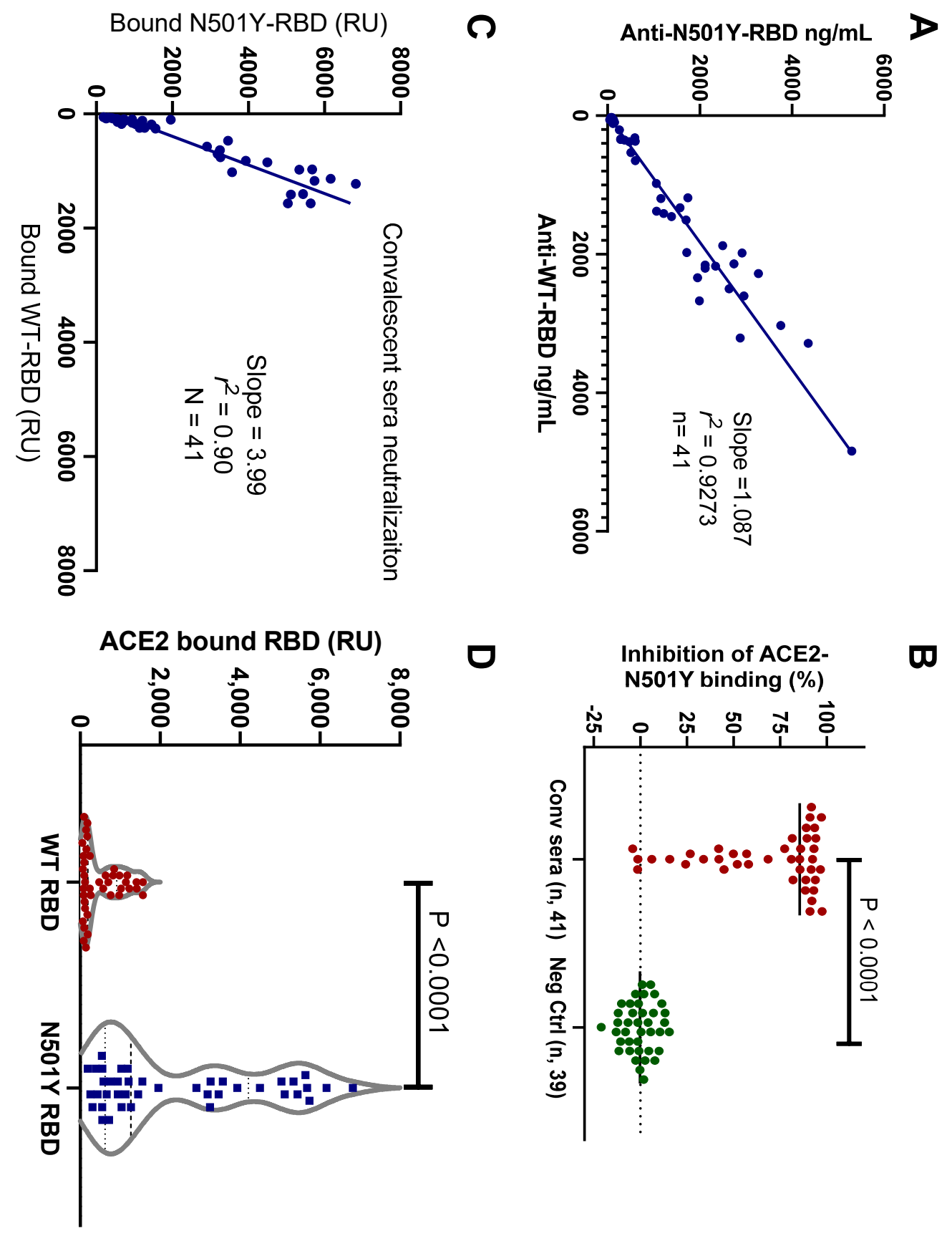




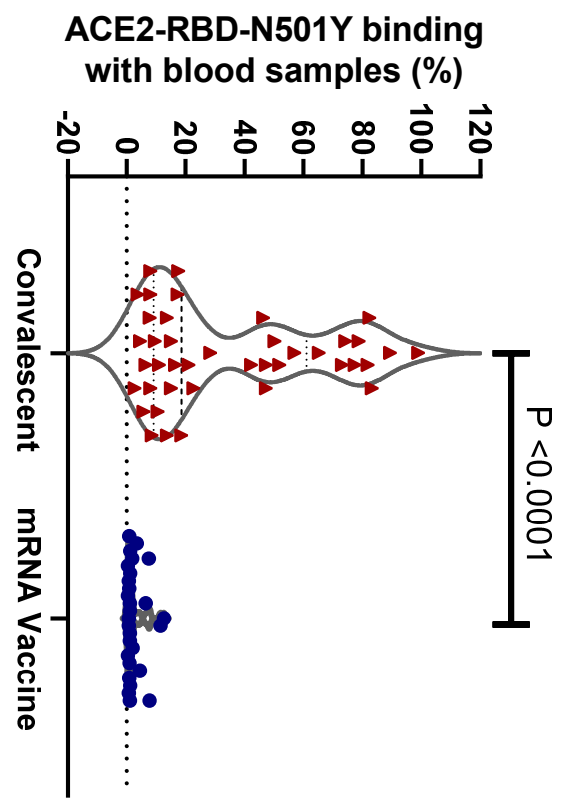

尺

ACE2-bound N501Y RBD \%

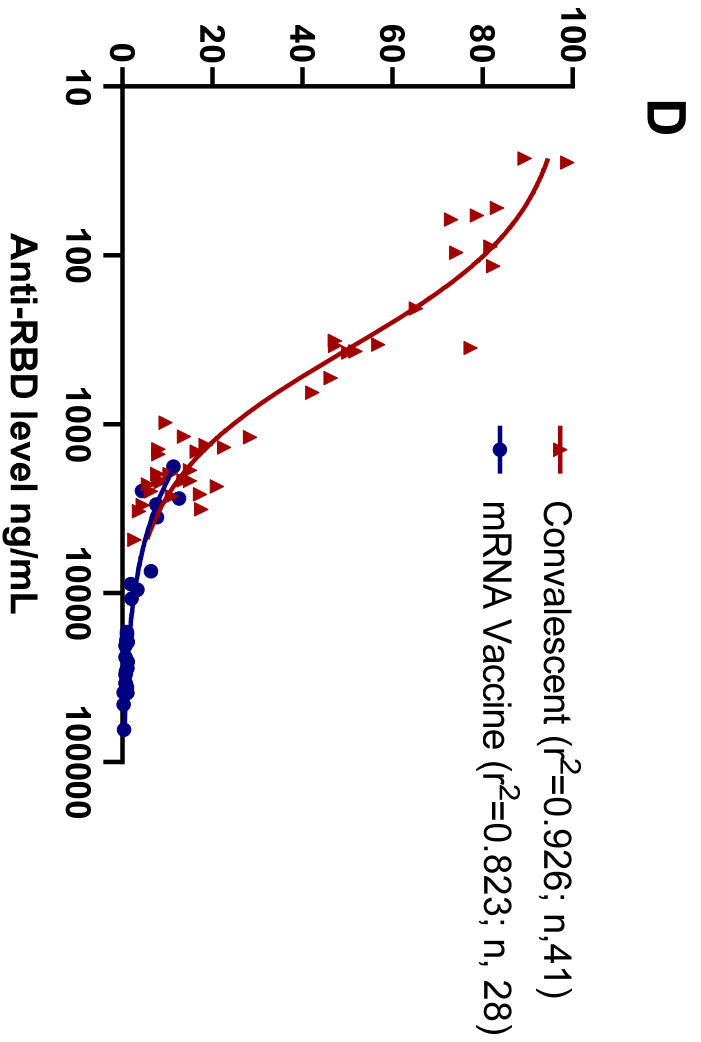

Inhibition of N501Y RBD-ACE2 binding (\%)

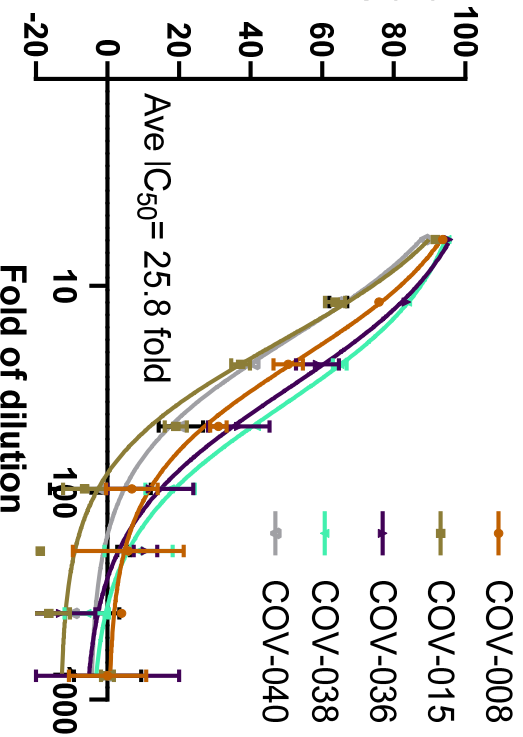

Inhibition of N501Y RBD-ACE2 binding (\%)

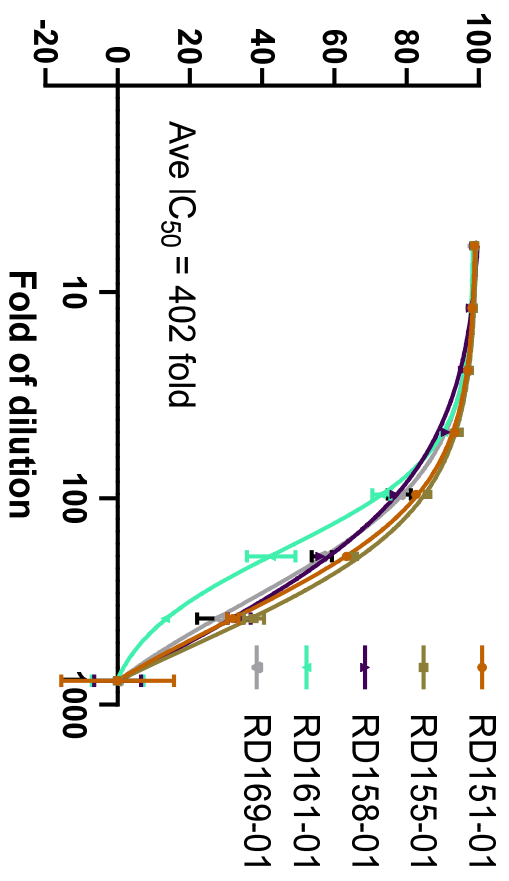




\section{Supplementary Files}

This is a list of supplementary files associated with this preprint. Click to download.

- SupplementarycombinedSR1.2.pdf 